\title{
Cyclosporine A-nanoparticles enhance the therapeutic benefit of adipose tissue- derived stem cell transplantation in a swine myocardial infarction model
}

\author{
This article was published in the following Dove Press journal: \\ International Journal of Nanomedicine \\ 10 December 2013 \\ Number of times this article has been viewed
}

\author{
Qiaoxiang Yin' \\ Zhiyong $\mathrm{Pei}^{2}$ \\ Heng Wang ${ }^{3}$ \\ Yusheng Zhao ${ }^{4}$ \\ 'Department of Geriatric Cardiology, \\ Chinese General Hospital of the \\ Air Force, ${ }^{2}$ Department of Geriatric \\ Cardiology, Beijing Military General \\ Hospital, ${ }^{3}$ Department of Neurology, \\ Chinese General Hospital of the \\ Air Force, ${ }^{4}$ Institute of Geriatric \\ Cardiology, Chinese PLA General \\ Hospital, Beijing, People's Republic of \\ China
}

\begin{abstract}
Treatment of myocardial infarction (MI) with adipose-derived stem cells (ASCs) has produced promising results. Cyclosporine $\mathrm{A}(\mathrm{CsA})$ inhibits apoptosis by preventing the opening of mitochondrial permeability transition pores. A CsA nanoparticle emulsion (CsA-NP) has lower toxicity and higher efficiency as compared to CsA. In this study, we hypothesized that a combination of ASCs and CsA-NP would enhance the therapeutic efficiency in a swine MI model. MI was induced in pig hearts by occlusion of the left anterior descending artery. The animals that survived MI were divided into four groups and 1 week later received intracoronary ASCs (ASCs, $n=6$ ), intracoronary culture media in combination with CsA-NP (CsA-NP, n=6), intracoronary ASCs in combination with CsA-NP (ASCs + CsA-NP, n=6), or remained untreated (control, $n=4$ ). Animals were sacrificed 8 weeks later and were evaluated for cardiac function by delayed-enhanced magnetic resonance imaging and immunohistopathology. We observed that the left ventricular ejection fraction (LVEF) was significantly increased in the ASCs + CsA-NP group, compared to the CsA-NP group (53.6\% $\pm 2.4 \%$ versus $48.6 \% \pm 1.5 \%, P<0.05)$, and the ASCs group $(53.6 \% \pm 2.4 \%$ versus $48.3 \% \pm 1.8 \%, P<0.05)$. More importantly, the infarct size was significantly smaller in the ASCs + CsA-NP group as compared to the CsA-NP group $\left(6.2 \pm 1.7 \mathrm{~cm}^{3}\right.$ versus $\left.9.1 \pm 3.4 \mathrm{~cm}^{3}, P<0.05\right)$ and the ASCs group $\left(6.2 \pm 1.7 \mathrm{~cm}^{3}\right.$ versus $7.5 \pm 0.6 \mathrm{~cm}^{3}$, $P<0.05)$. These findings were further confirmed by analysis of the expression of cardiomyocyte markers, myosin heavy chain ( $\alpha$-actinin) and troponin T. In addition, the CsA-NP + ASCs treatment promoted neovascularization $(P<0.05)$ and inhibited cardiomyocyte apoptosis $(P<0.01)$ compared to the control group. This study demonstrates that CsA-NP enhanced the therapeutic benefits of ASCs transplantation for MI.
\end{abstract}

Keywords: cyclosporine A nanoparticle emulsion, cyclosporine A, adipose tissue-derived stem cells, myocardial infarction

\section{Introduction}

Although the use of reperfusion and pharmacological therapies has prolonged survival and increased the quantity of salvageable tissue following myocardial infarction (MI), this condition is still a degenerative disease and shows a peak in cell death due to the irreversible loss of cardiomyocytes and the process of tissue remodeling, which eventually leads to heart failure. Recently, mesenchymal stem cell-based therapies have become increasingly recognized for their potential to repair the damaged myocardium after MI. Among these, adipose tissue-derived stem cells (ASCs) are an attractive source of mesenchymal stem cells due to multilineage differentiation potential of this
Correspondence: Qiaoxiang Yin Department of Geriatric Cardiology, Chinese General Hospital of Air Force, Fuchenglu, Beijing I00I42, People's Republic of China Tel +8613681377055 $\mathrm{Fax}+8616841009968161$ Email zmyyqx-2004II29@I63.com 
cell type, the abundance of subcutaneous adipose tissue, the simple surgical procedure involved, and a higher stem cell yield. ${ }^{1,2}$ Despite the structural and functional benefits derived from stem cell-based therapies for MI, limited survival of transplanted cells has been observed due to apoptosis and necrosis. ${ }^{3}$ Thus, the challenge in cardiac cell therapy is associated with the strengthening of donor cells to allow survival in the harsh microenvironment of the infarcted heart.

In addition to its well-known immunosuppressive properties, cyclosporine A (CsA) inhibits apoptosis by preventing the opening of mitochondrial permeability transition pores (mPTPs), and some reports indicate that it can limit ischemia-reperfusion injury. ${ }^{4}$ Interest in its clinical use has also recently been piqued by a report demonstrating CsA effectiveness in limiting the infarct size in MI patients. ${ }^{5}$ The results from experimental studies and clinical applications have generally been encouraging. However, the severe adverse effects of long-term CsA usage have limited its success in clinical use. ${ }^{6}$ It is reasonable to manipulate CsA to improve drug targeting, lower toxicity, and increase efficiency and convenience.

CsA has been studied in many pharmaceutical formulations due to its absorption and bioavailability issues. ${ }^{7}$ Nanoparticles (NP) may improve the biodistribution of drugs and increase their half-lives, leading to enhanced therapeutic effects. ${ }^{8}$ Using the targeting drug delivery system utilized in cancer chemotherapy, we prepared a CsA nanoparticle emulsion (CsA-NP) in this study. We examined the inhibition of apoptosis and the protective effects of CsA-NP on autologous ASC transplantation after MI in a swine model. Cardiac function was evaluated by delayed-enhanced magnetic resonance imaging (DE-MRI) and immunohistopathology.

\section{Materials and methods}

\section{Ethics statement}

This study was carried out in strict accordance with the recommendations in the Guide for the Care and Use of Laboratory Animals of the National Institutes of Health and the State Science and Technology Commission, People's Republic of China. The protocol was approved by the ethics committee of the Chinese General Hospital of the Air Force in Beijing (permit number: 090457). All animals were from a pig farm maintained by the Northeast Agricultural University, Chinese Academy of Agricultural Sciences Harbin branch and were allowed to acclimatize for at least 7 days prior to experimentation. All animals in this experiment were housed in individual cages under light-controlled conditions and at room temperature (Animal Supplier Center of
Chinese PLA General Hospital, Beijing, People's Republic of China). All surgery was performed under sodium pentobarbital anesthesia, and all efforts were made to minimize suffering. The pigs were sacrificed by bleeding out through the cervical artery after cell transplantation at 8 weeks. Some tissue samples were removed from animals and immediately snap-frozen in liquid nitrogen and stored at $-80^{\circ} \mathrm{C}$ until subsequent analysis.

\section{Experimental animals}

Chinese Bama swine ( $\mathrm{n}=35$ ) of both sex, weighing $20-30 \mathrm{~kg}$, were used in this study. Of the 35 animals randomized in the study, nine died from ventricular fibrillation upon initial MI induction, and four died in the postoperative period. Complete follow-up data were obtained from 22 animals, which were randomly assigned to four groups: control (control, $n=4)$, intracoronary ASCs alone (ASCs, n=6), CsA-NP plus intracoronary culture medium phosphate buffered saline (PBS) without cells (CsA-NP, $n=6$ ), and CsA-NP plus intracoronary ASCs (CsA-NP + ASCs, n=6). Six additional swine were serially scanned before cell transplantation and 8 weeks after cell transplantation to account for growth and biological agerelated changes in left ventricle (LV) volume and mass.

\section{Preparation of CsA-NP}

An oil-in-water emulsification-diffusion method was used to prepare the CsA-NP intravenous emulsion in the present study, using Lipoid E 80 and poloxamer 188 (Shenyang Pharmaceutical University, Nanjing, People's Republic of China) as emulsifiers and Lipoid medium-chain-triglyceride (Shenyang Pharmaceutical University) as the oil phase. The products were evaluated by measuring drug loading by high performance liquid chromatography, and the product particle size was evaluated by dynamic light scattering. The pharmacokinetic behavior of the CsA-NP was studied following intravenous administration to rats and compared with that of CsA (Sandimmune ${ }^{\circledR}$; East Hanover, NJ, USA). The safety of CsA-NP was evaluated by the following tests: abnormal toxicity test, blood vessel irritation test, and hemolysis test.

\section{Cell preparation and labeling}

Subcutaneous adipose tissue was harvested from the inguinal area. Adipose tissue was minced and processed as described. ${ }^{1,2}$ After 48-72 hours, non-adherent cells were discarded by washing with PBS. During the entire period of culture, growth medium was changed three times a week. After reaching 80\%-90\% of confluence, ASCs were detached for further expansion and experiments. 
ASCs were characterized by examining cell surface marker expression by flow cytometry with antibodies against CD29, CD31, CD34, CD44, CD45, CD90, CD105, and HLA-DR (eBioscience, San Diego, CA, USA) according to the manufacturer's protocols. The cells were washed twice with PBS and stained with 4',6-diamidino-2-phenylindole (DAPI) (Sigma-Aldrich, St Louis, MO, USA) according to the manufacturer's protocol on the day of transplantation. The ASC concentration was adjusted to $1 \times 10^{7}$ cells $/ \mathrm{mL}$. After labeling, cells were washed with PBS, resuspended in $100 \mathrm{~mL}$ saline, and kept on ice.

\section{Creation of the MI model and cell transplantation}

The swine were intubated under general anesthesia and positive pressure ventilation was maintained. MI was induced by placing an angioplasty catheter into the middle segment of the left anterior descending artery and inflating it for 90 minutes, followed by reperfusion. ST-T wave changes in the electrocardiogram (ECG) and follow-up coronary arteriographies were recorded to confirm occlusion of the vessel. During the procedure, the intravenous antiarrhythmic medication administered consisted of amiodarone at 25-75 $\mathrm{mg}$ per bolus during ventricular arrhythmias. Anticoagulant doses of heparin were given intravenously (bolus of 3,000 U) at the beginning of infarction induction, followed by $1,000 \mathrm{U} /$ hour until the end of the surgery. Aspirin and oral $\beta$-blockers were given on a daily basis from 1 day before MI until sacrifice. One week after MI, ASCs $\left(4 \times 10^{7}\right.$ cells $)$ or PBS were delivered by intracoronary injection into the left anterior descending artery. CsA-NP and CsA-NP + ASCs groups received intravenous injection of $2.0 \mathrm{mg} / \mathrm{kg}$ CsA-NP before reperfusion of the MI and before ASCs transplantation. The CsA-NP dose was chosen based on our experiences in vitro. ${ }^{9}$

\section{DE-MRI}

To test the effects on cardiac function after transplantation, cardiac MRI (1.5T HD Signa Excite; GE Healthcare, Piscataway, NJ, USA) with ECG triggering was performed before cell transplantation and 8 weeks after cell transplantation. Global function was assessed by breath-hold cine MRI in the short, vertical, and horizontal long axes. In the short axis, the LV was completely encompassed by contiguous $6 \mathrm{~mm}$ thick slices. Delayed enhancement imaging was applied for infarct assessment after injection of $0.15 \mathrm{mmoL} / \mathrm{kg}$ of gadopentetate dimeglumine using a phase-sensitive inversion recovery sequence (repetition time/echo time, 11/4.45 millisecond; flip angle, $20^{\circ}$; inversion time, 250 milliseconds; bandwidth, $62.5 \mathrm{KHz}$; field of view, $26 \times 26 \mathrm{~cm}$; slice thickness, $6.0 \mathrm{~mm}$; spacing, $0.5 \mathrm{~mm}$; matrix, $160 \times 192$; number of excitations, 3 ). All images were analyzed on a Mass 6 workstation (HD Signa Excite; GE Healthcare).

\section{Morphologic assessment and identification of the transplanted cells}

Eight weeks after cell transplantation, animals were returned for DE-MRI and then euthanized. The hearts were immediately excised and prepared for morphologic assessment and histological analysis. Each heart was excised, and the LV was isolated and fixed in a $20 \%$ gelatin solution for 20 minutes. After fixation, the LV was sectioned into eight 2-3 mm transverse slices along the short-axis plane. The thickness of each slice was measured with a digital micrometer, and all slices were imaged. Samples of infarct tissue, border zones, and noninfarcted myocardium from every slice were excised and flash-frozen in liquid nitrogen or fixed in $10 \%$ formalin, embedded, and cut in $5 \mu \mathrm{m}$ sections. To assess cardiogenic transdifferentiation of the engrafted ASCs, the following antibodies were used to identify the transplanted cells in the heart: mouse anti- $\alpha$-actin (1:50) and mouse anti-troponin $\mathrm{T}$ (1:50) (Abcam, Cambridge, UK).

\section{Analyses of vascular density}

The vascular density was assessed by staining for von Willebrand factor using 3,3'-diaminobenzidine tetrahydrochloride as a chromogen. The secondary antibody was biotinylated goat anti-rabbit Immunoglobulin G (Boster Biological Engineering Co., Beijing, People's Republic of China). The density and size of blood vessels were evaluated in six histological sections per animal from the infarct tissue and border zone. Pictures were taken under light microscopy at $400 \times$ magnification in six random fields $\left(0.1 \mathrm{~mm}^{2}\right)$ per slide. Only vessels with a diameter equal to or below a $10-100 \mu \mathrm{m}$ range on a perpendicular cut were counted with Image Pro Plus 4.5 software (Media Cybernetics, Rockville, MD, USA).

\section{Counts of apoptotic myocardial cells}

The in situ terminal deoxynucleotidyl transferase mediated dUTP nick end-labeling (TUNEL) method was used to detect myocardial cell apoptosis, according to the manufacturer's protocol (Santa Cruz Biotechnology Co., Ltd, Santa Cruz, CA, USA). Ten zones were randomly chosen from each slide, and the number of positive apoptotic cells per 100 cells in each zone was determined. The average number was taken to be the positive rate of apoptotic cell death. 


\section{Statistical analysis}

Data were expressed as mean \pm standard deviation. The Student's unpaired $t$-test was used to compare quantitative variables among the four study groups, while the Mann-Whitney $U$ test was used when data were not normally distributed. Analysis of variance (ANOVA) was used for multigroup comparison. The SPSS version 17.0 software program (IBM Corporation, Armonk, NY, USA) was used for statistical analysis, and $P<0.05$ was considered statistically significant.

\section{Results}

Characteristics of ASCs before implantation

In vitro cell surface marker expression was used for characterization of the ASCs. Flow cytometry revealed that the cultured swine ASCs were positive for CD29 $(99.06 \% \pm 0.30 \%)$, CD44 (98.20\% $\pm 0.30 \%)$, CD90 (97.40\% $\pm 0.40 \%$ ), and CD105 $(98.30 \% \pm 0.44 \%)$ and negative for CD31 $(2.06 \% \pm 0.06 \%)$, CD34 (2.34\% $\pm 0.33 \%), C D 45$ (1.98\% $\pm 0.08 \%$ ), and HLA-DR $(1.66 \% \pm 0.08 \%)$ (Figure 1). The characteristics of surface
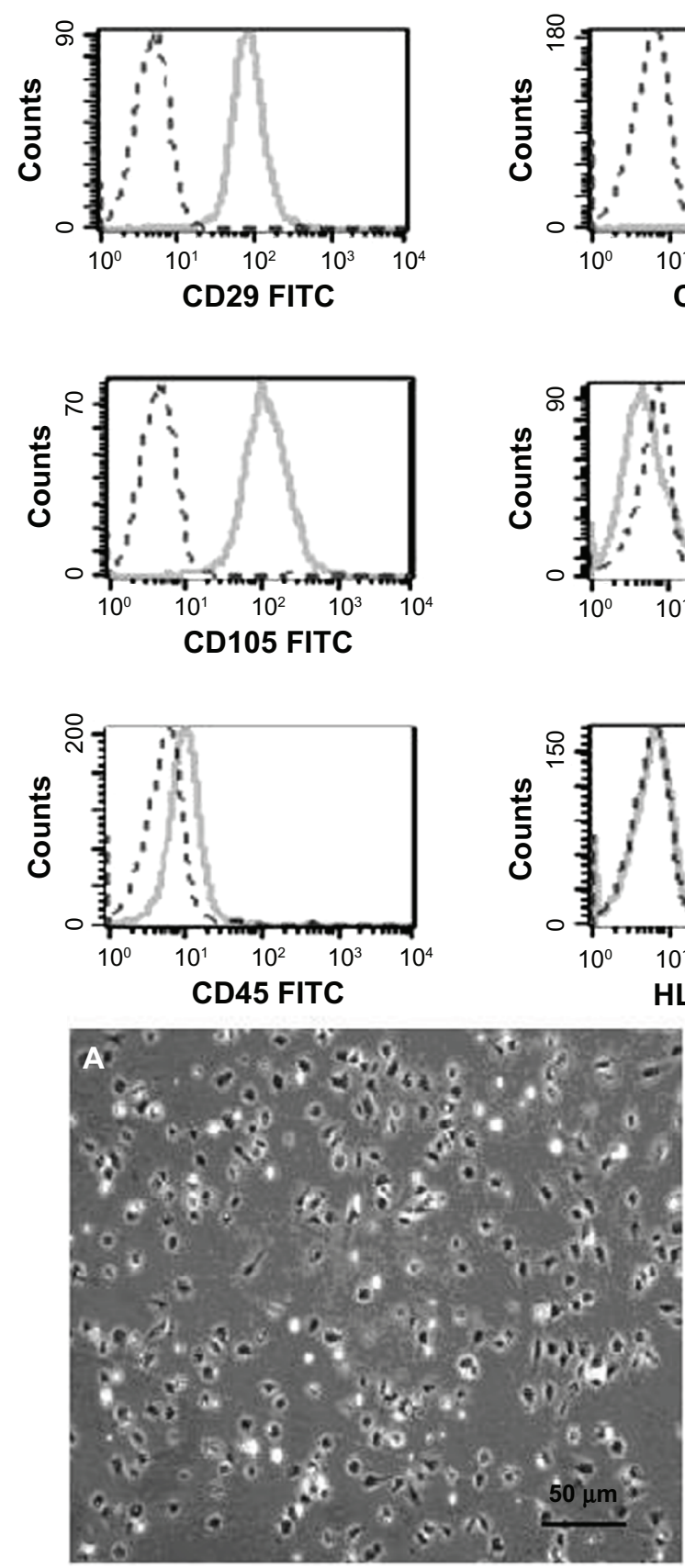
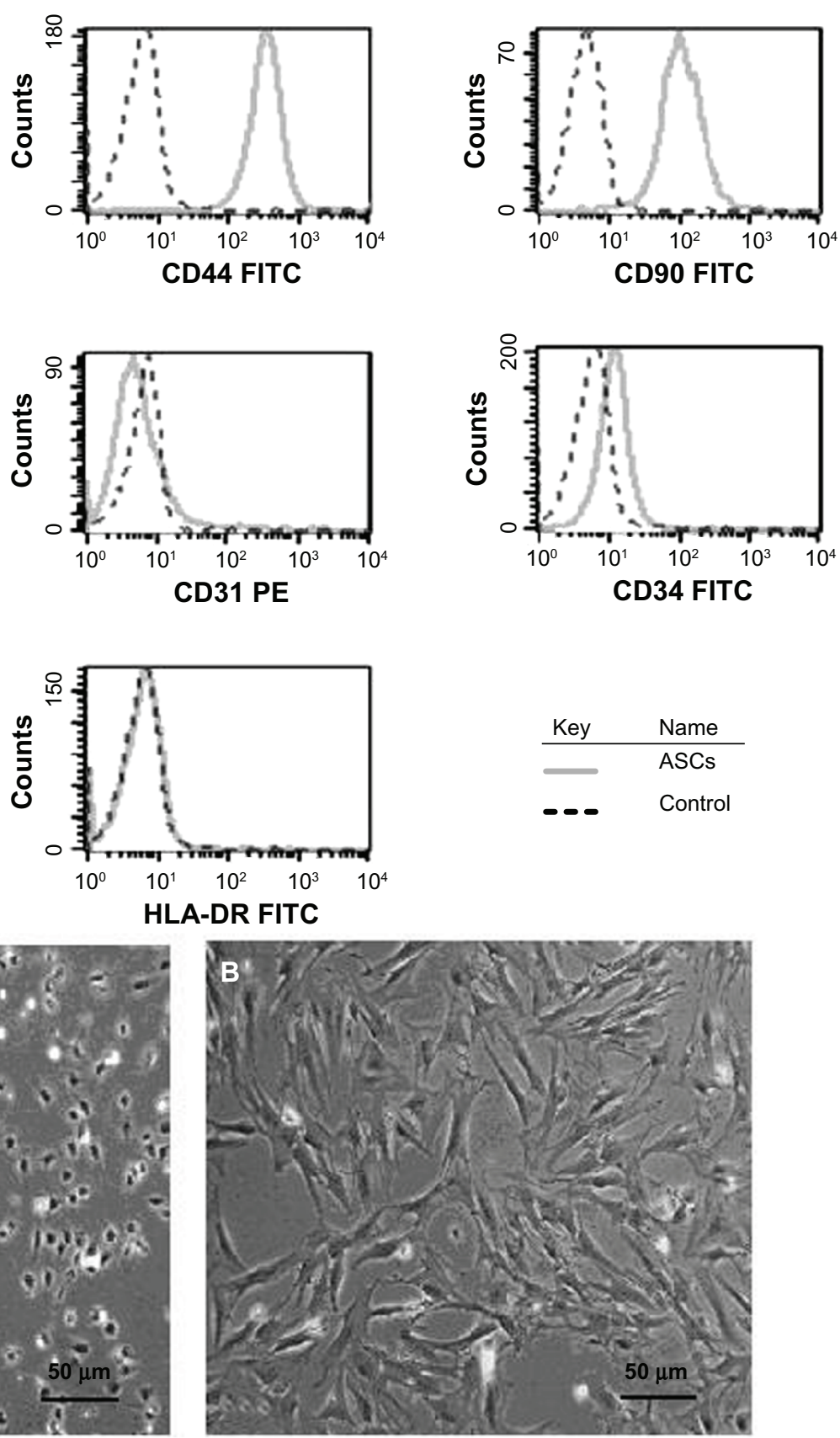

Figure I Flow cytometry assessment of cell surface marker expression and characterization of ASC morphology in vitro.

Notes: Morphology of primary ASCs 24 hours after seeding (A) (original magnification I00x). Morphology of ASCs after 7 days of culture (B) (original magnification I00x). Abbreviations: ASC, adipose tissue-derived stem cell; FITC, fluorescein isothiocyanate; PE, phycoerythrin. 
marker expression of the ASCs found in this study indicated the ASCs expressed the mesenchymal cell specific markers, which were in agreement with those previously reported by others. ${ }^{10}$

\section{DE-MRI measurements and data analysis}

Before cell transplantation, DE-MRI showed that the average left ventricular ejection fraction (LVEF) equivalently and significantly decreased in all groups compared with the values in healthy pigs (data not shown). However, 8 weeks after cell transplantation, LVEF significantly increased in the CsA-NP + ASCs group compared to the control group $(53.6 \% \pm 2.4 \%$ versus $46.2 \% \pm 3.9 \%, P<0.01)$, the CsA-NP group (53.6\% $02.4 \%$ versus $48.6 \% \pm 1.5 \%, P<0.05)$, and the ASCs group $(53.6 \% \pm 2.4 \%$ versus $48.3 \% \pm 1.8 \%, P<0.05)$ (Figure 2). There was no statistical difference between the CsA-NP group and the ASCs group $(P>0.05)$. In contrast, left ventricular end-diastolic volume significantly decreased in the CsA-NP + ASCs group compared to the control group $(52.8 \pm 3.3 \mathrm{~mL}$ versus $61.6 \pm 7.8 \mathrm{~mL}, P<0.05)$. A similar trend was not seen for left ventricular end-systolic volume (LVESV); there were no statistical differences among the four groups. More importantly, infarct size was significantly smaller in the CsA-NP + ASCs group compared with the control group $\left(6.2 \pm 1.7 \mathrm{~cm}^{3}\right.$ versus $\left.9.8 \pm 2.4 \mathrm{~cm}^{3}, P<0.01\right)$, the CsA-NP group $\left(6.2 \pm 1.7 \mathrm{~cm}^{3}\right.$ versus $\left.9.1 \pm 3.4 \mathrm{~cm}^{3}, P<0.05\right)$, and the ASCs group $\left(6.2 \pm 1.7 \mathrm{~cm}^{3}\right.$ versus $\left.7.5 \pm 0.6 \mathrm{~cm}^{3}, P<0.05\right)$. The left ventricular mass index was $72.2 \pm 2.6 \mathrm{~g} / \mathrm{m}^{2}, 70.8 \pm 2.3 \mathrm{~g} / \mathrm{m}^{2}, 69.2 \pm 3.8 \mathrm{~g} /$ $\mathrm{m}^{2}$, and $67.6 \pm 2.2 \mathrm{~g} / \mathrm{m}^{2}$ for the control, ASCs, CsA-NP, and CsA-NP + ASCs group, respectively, without differences between groups. LV wall thickness decreased in the ASCs group compared to the control group $(-21.8 \% \pm 3.6 \%$ versus $-26.5 \% \pm 1.8 \%, P<0.05)$, but the change was not seen in the
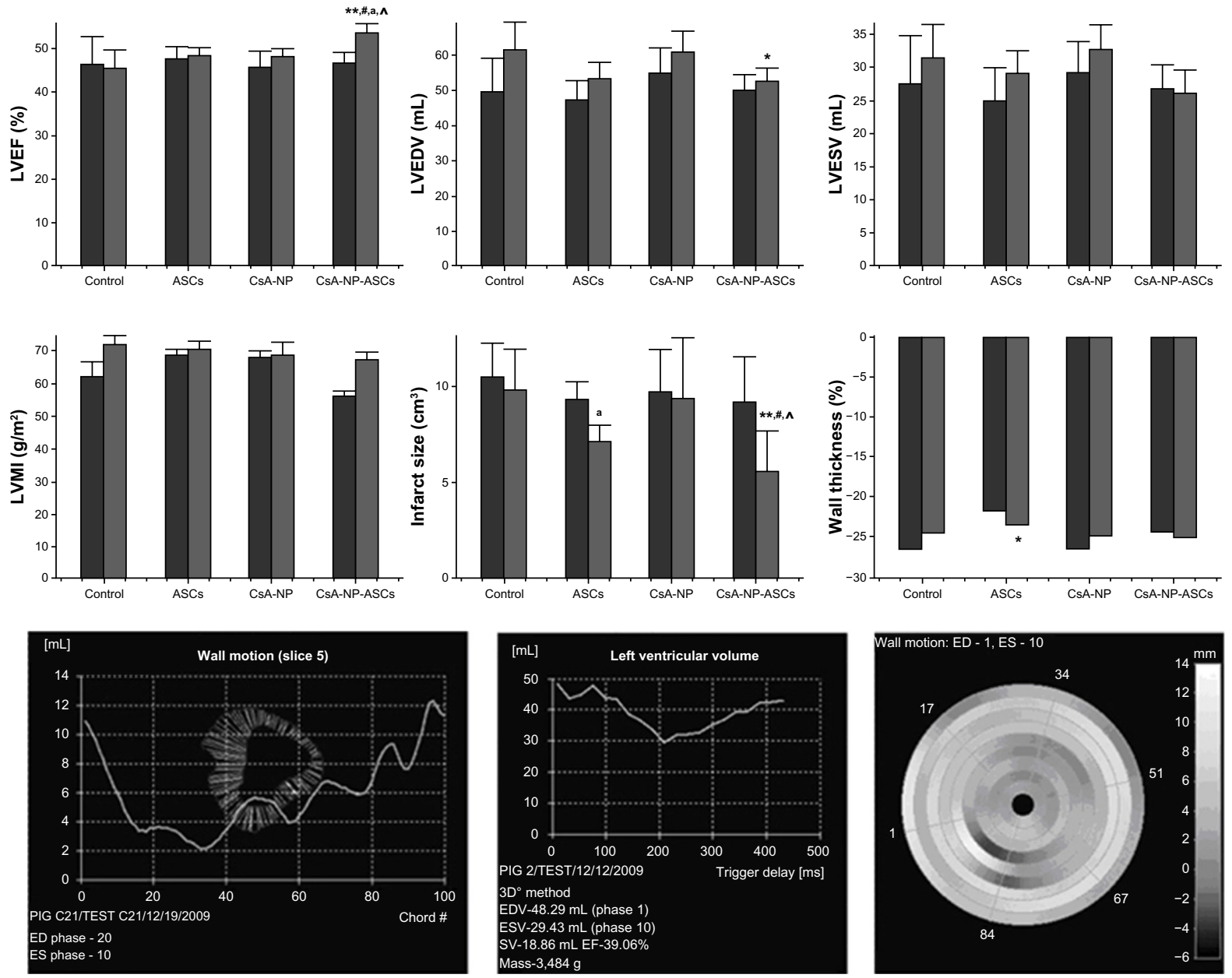

Figure 2 Left ventricular function and geometry assessment by MRI.

Notes: Baseline versus endpoint, $\square$ baseline $\square$ endpoiont. $* P<0.05$ versus control; $* * P<0.0$ I versus control; $\# P<0.05$ versus CsA-NP; $\wedge P<0.05$ versus ASCs; ASC; ${ }^{a} P<0.0$ I baseline versus endpoint.

Abbreviations: ASCs, adipose tissue-derived stem cells; CsA-NP, cyclosporine A nanoparticle emulsion; ED, end-diastolic; EDV, end-diastolic volume; EF, ejection fraction; ES, end-systolic; ESV, end-systolic volume; LVEDV, left ventricular end-diastolic volume; LVEF, left ventricular ejection fraction; LVESV, left ventricular end-systolic volume; LVMI, left ventricular mass index; SV, stroke volume. 

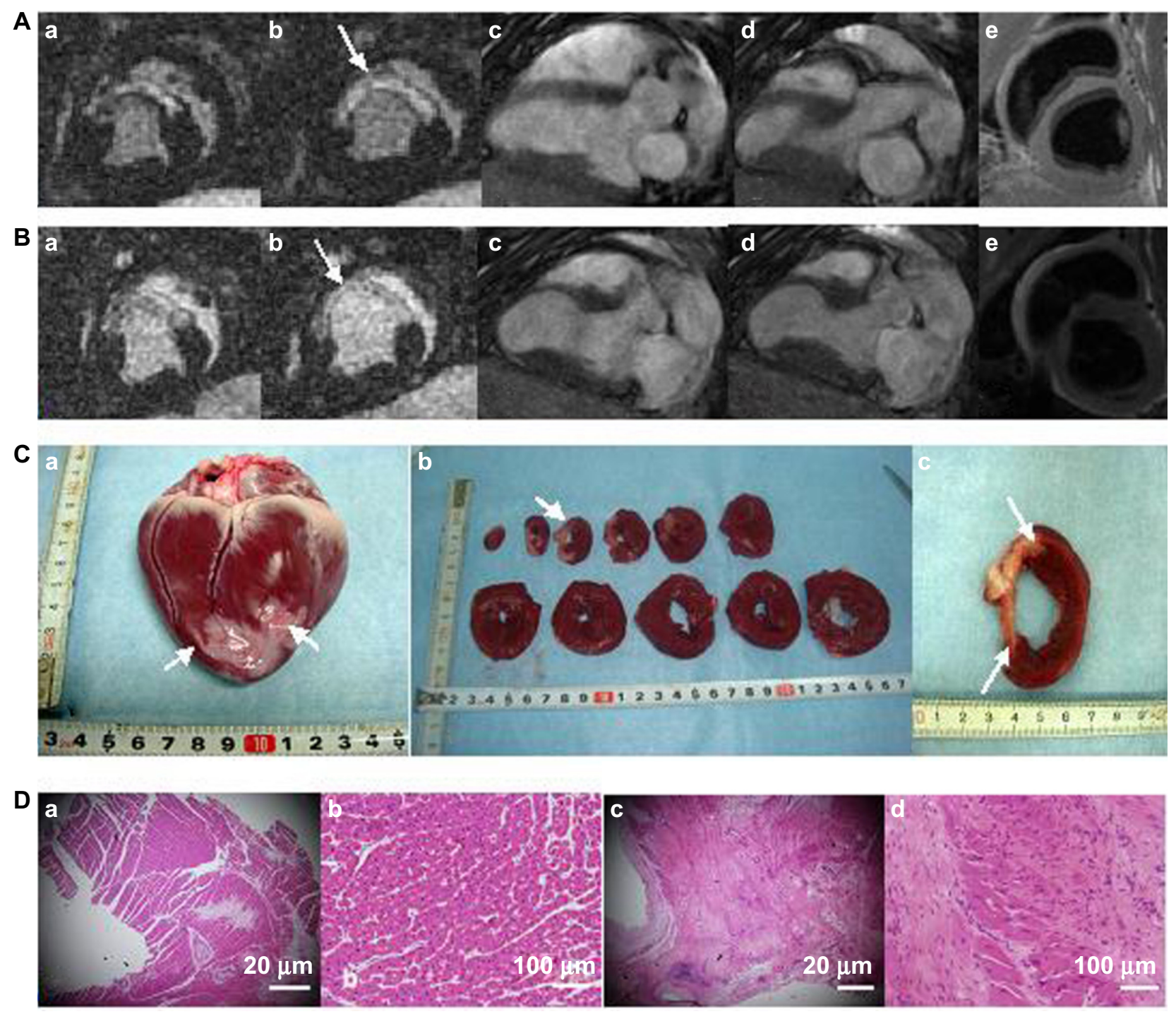

Figure 3 Representative in vivo DE-MRI and ex vivo histological samples from CsA-NP+ASCs-treated hearts.

Notes: Before cell transplantation (A) DE- MRI with high signal intensity in the sectional short-axis (a,b) view demonstrate infarct myocardial viability (arrows), long-axis in the diastolic (c) and systolic (d) and the black-blood T2-weighted (e); 8 weeks after cell transplantation (B) DE-MRI with high signal intensity in the sectional short-axis $(a, b)$ view demonstrate infarct myocardial viability (arrows), long-axis in the diastolic (c) and systolic (d) and the black-blood T2-weighted (e). Corresponding photographs of histomorphometry (C) demonstrate the porcine heart (a), cross-sectional slices (b) and one representative slice (c) (the arrows indicate pale areas are the infarction myocardial areas). Pathological changes were observed by HE staining (D), infarct myocardium tissue (a and b); normal myocardium tissue (c and d).

Abbreviations: ASCs, adipose tissue-derived stem cells; CsA-NP, cyclosporine A nanoparticle emulsion.

CsA-NP + ASCs group and the CsA-NP group. Figure 3 shows the morphologic assessment in vivo by DE-MRI and the ex vivo sample histology. In vivo findings by DE-MRI and the ex vivo sample histology are in accord with each other, indicating consistent conclusion from these methods.

\section{ASC engraftment and phenotype after administration}

Eight weeks after cell transplantation, immunofluorescence staining of frozen sections showed DAPI-labeled nuclei in the left ventricular myocardium distinguishing the ASCs group and CsA-NP + ASCs group (Figure 4). The distribution of DAPI-labeled cells demonstrated survival of transplanted cells. Many of the DAPI-labeled cells stained positively for cardiac-specific troponin $\mathrm{T}$ and $\alpha$-sarcomeric actin, suggesting that the transplanted cells differentiated into myogenic cells. As expected, we observed nearly a doubling in the number of engrafted cells (blue, DAPI-labeled nuclei positive cells) in the CsA-NP + ASCs group compared to the ASCs alone group $\left(29 \pm 6.8 \mathrm{cells} / \mathrm{cm}^{2}\right.$ versus $15 \pm 8.6 \mathrm{cell} / \mathrm{s} / \mathrm{cm}^{2}$, $P<0.05$ ), indicating that CsA-NP effectively improved survival of the transplanted cells.

\section{Capillary density}

To evaluate whether CsA-NP + ASC and ASC transplantation after MI were associated with enhanced neovascularization, 

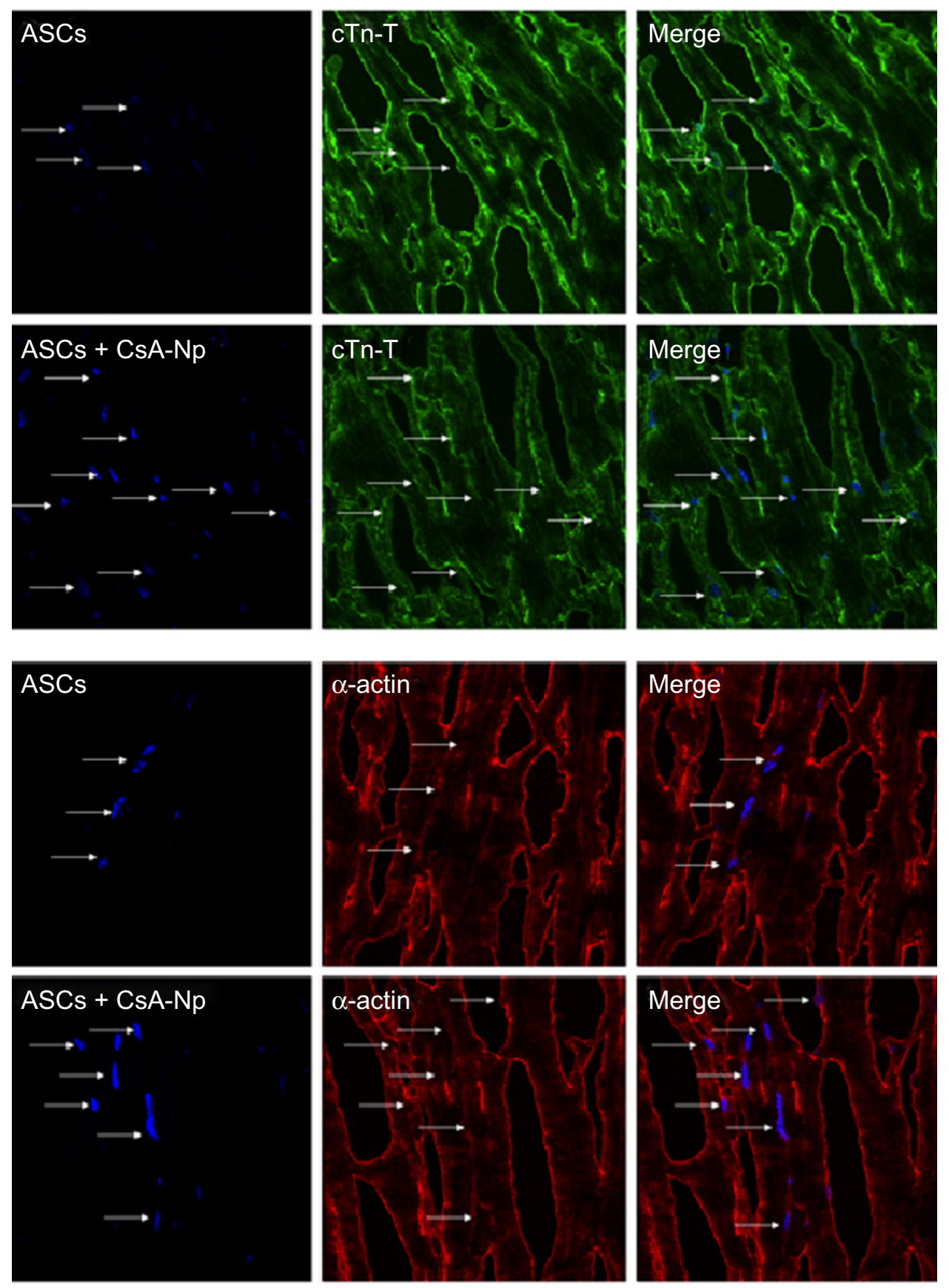

Figure 4 Immunofluorescence analysis of the engraftment and differentiation of DAPI-labeled cells in CsA-NP + ASCs- and ASCs-treated hearts.

Notes: Eight weeks after cell transplantation in vivo, cells were stained for troponin T and $\alpha$-actinin, and nuclei were stained with DAPI. Merge imaging is shown (magnification 600x).

Abbreviations: $\alpha$-actin, $\alpha$-actinin; ASCs, adipose tissue-derived stem cells; CsA-NP, cyclosporine A nanoparticle emulsion; cTn-T, troponin T; DAPI, 4',6-diamidino-2phenylindole.

vascular density was determined on von Willebrand factor-stained sections from the infarct core, border zone, and remote myocardium (Figure 5). No differences were seen among the four groups in the infarct remote myocardium. However, in the infarct core, the vascular density was significantly higher in the CsA-NP + ASCs group than in control group $\left(43 \pm 15 / \mathrm{mm}^{2}\right.$ versus $\left.36 \pm 10 / \mathrm{mm}^{2}, P<0.05\right)$. No differences were seen among the control group, the ASCs group, and the CsA-NP group $(P>0.05)$. There were no statistical differences among the four groups in the infarct border zone.

\section{Myocardial cell apoptosis}

The apoptotic index of myocardial cells in the CsA-NP+ASCs group, ASCs group, and CsA-NP group were significantly 

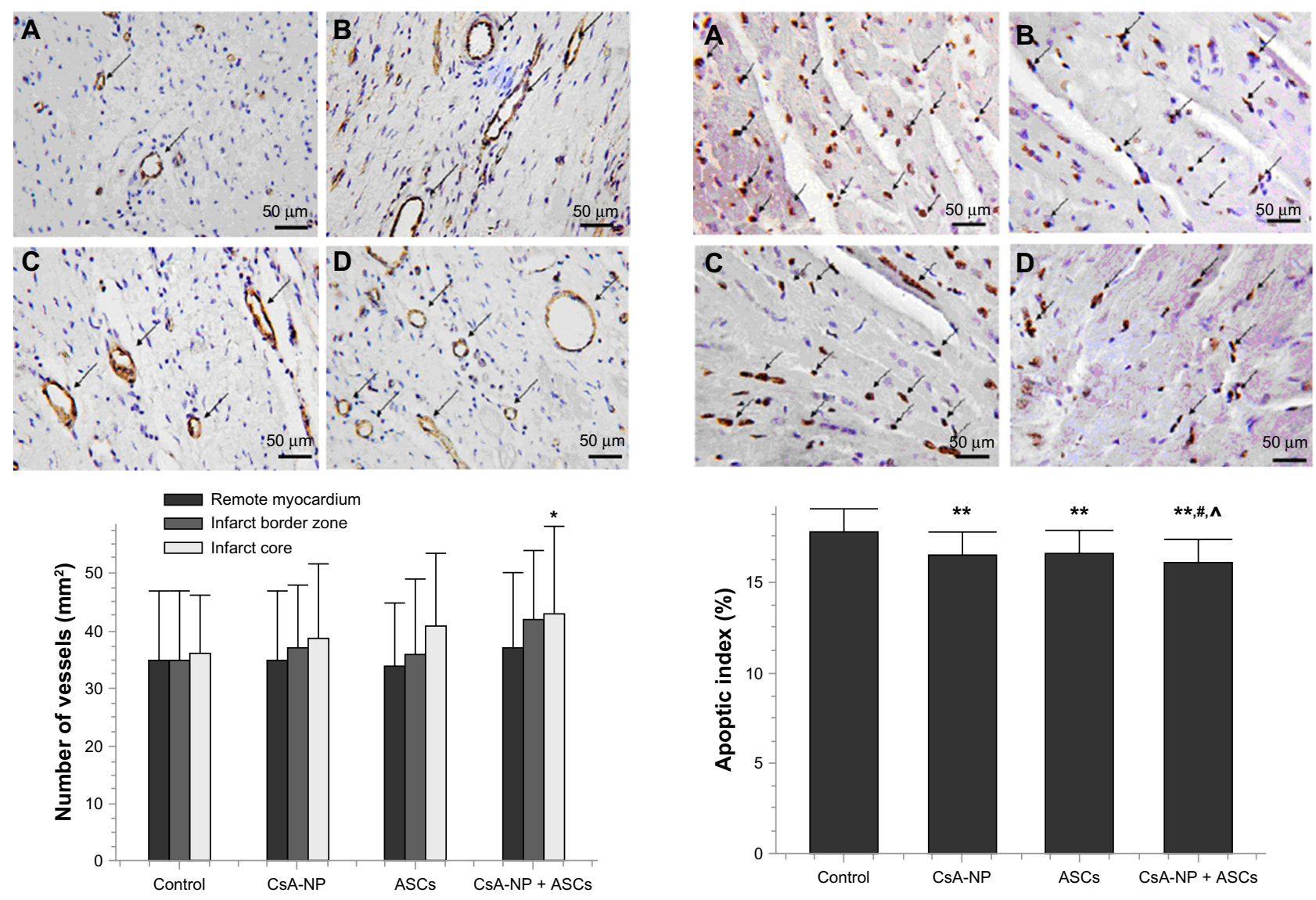

Figure 5 Microphotographs representing the comparative results of vascular density staining for von Willebrand factor in all groups.

Notes: (A) control; (B) CsA-N; (C) ASCs; (D) CsA-NP+ASCs. Large vessels are indicated by arrowheads. The CsA-NP + ASCs-treated group in the infarct zone show a higher capillary density compared with the control groups. ${ }^{*} P<0.05$ for CsA-NP + ASCs versus control.

Abbreviations: ASCs, adipose tissue-derived stem cells; CsA-NP, cyclosporine A nanoparticle emulsion.

lower than in controls $(P<0.01)$. However, the apoptotic index of myocardial cells in the CsA-NP + ASCs group was significantly lower than that in the CsA-NP group ( $16.12 \pm 1.27$ versus $16.58 \pm 1.26, P<0.05)$ and in the ASCs group $(16.12 \pm 1.27$ versus $16.66 \pm 1.29, P<0.05)$. There were no differences between the ASCs and CsA-NP groups (Figure 6).

\section{Discussion}

This was the first study to evaluate the feasibility and effectiveness of the use of ASCs in combination with CsA-NP (CsA-NP + ASCs) in a clinically relevant model of infarction. Compared with ASCs transplantation alone, ASCs in combination with CsA-NP further repaired the damaged myocardium, improved cardiac function, and prevented left ventricular dilatation after cell transplantation. In the present study, serial sections of the histological samples showed that DAPI-labeled cells expressed cardiomyocyte-specific markers (troponin $\mathrm{T}, \alpha$-actinin). Histological samples also

Figure 6 Myocardial cell apoptosis by TUNEL in all groups.

Notes: (A) control; (B) CsA-N; (C) ASCs; (D) CsA-NP+ASCs. The apoptotic index was significantly lower in CsA-NP + ASCs group; $* * P<0.01$ versus control; $\# P<0.05$ CsA-NP + ASCs versus CsA-NP; $\wedge P<0.05$ CsA-NP + ASCs versus ASCs. Abbreviations: ASCs, adipose tissue-derived stem cells; CsA-NP, cyclosporine A nanoparticle emulsion; TUNEL, terminal deoxynucleotidyl transferase dUTP nick end-labeling.

showed that ASCs in combination with CsA-NP promoted neovascularization and reduced myocardial cell apoptosis. These additional cells increased scar thickness and prevented scar expansion, which may be the mechanism underlying the CsA-NP + ASCs combination-induced improvement in cardiac function. Supporting this hypothesis, DE-MRI showed that the CsA-NP + ASCs combination increased regional perfusion and wall motion and prevented scar thinning and ventricular dilatation. Importantly, this rapid improvement in cardiac function suggests that the functional improvement was related to cardiac transdifferentiation of the ASCs or myocardial regeneration. Indeed, the present study suggests that CsA-NP further enhanced the therapeutic benefits of ASCs transplantation into the infarcted myocardium.

Pharmacokinetic evaluation of CsA-NP in rats showed that the area under the curve increased significantly $(P<0.05)$ and the clearance rate was decreased $(P<0.05)$, which illustrated that the CsA-NP could be maintained at a relatively 
high concentration in blood and therefore enhance the antiapoptosis effect. The safety results showed that CsA-NP induced neither serious intravenous toxicity nor undesirable irritation to the veins. Moreover, no hemolysis was observed in the common test tube method in vitro. The plasma CsA-NP concentration released in vivo remained stable for 5 days. The preparation technique used produced an optimized stable formulation with uniform particle size distribution, with the dimension of $50 \%$ of the emulsion droplets being less than $162 \mathrm{~nm}$. Thus, the intravenous CsA-NP emulsion is stable and represents a new type of drug delivery system.

There were several possible mechanisms for the improvement of ASCs transplantation benefits via CsA-NP. In addition to its well-known immunosuppressive properties, CsA is a potent inhibitor of the opening of the MPTP by binding to cyclophilin D in the mitochondrial matrix. ${ }^{11}$ The $\mathrm{mPTP}$ opens under a variety of conditions related to mitochondrial dysfunction, such as calcium overload and excessive production of reactive oxygen species. ${ }^{12}$ The opening of mPTPs in the inner mitochondrial membrane results in the collapse of the membrane potential and caspase- 3 (the final common effector protease mediating apoptosis signaling) activation, and these metabolic alterations may lead to cardiomyocyte apoptosis. In fact, CsA has been shown to induce comparable protection in acute MI (AMI) patients. ${ }^{13}$

Although the use of reperfusion and pharmacological therapy has dramatically increased the rate of survival after MI, the irreversible loss of cardiomyocytes due to necrosis and apoptosis and the process of tissue remodeling typically leads to heart failure at a later point. Recently, accumulating evidence suggests that implantation of stem cells into damaged myocardia could regenerate cells within the infarct and prevent heart failure. Hence, ASCs represent a promising source of adult stem cells for cardiovascular repair. ${ }^{14}$ The similarities between bone marrow-derived cells and ASCs suggest the potential of adipose tissue to act as an alternative for repairing damaged myocardium. ${ }^{15}$ ASCs are able to differentiate into multiple cell lineages including cardiomyocytes. ASCs can effectively improve LVEF in animal models of AMI and chronic MI. ${ }^{16}$ According to a small, first-of-its-kind study, ASCs can be safely obtained and infused inside the hearts of patients following an acute heart attack. ${ }^{17}$ However, it is widely recognized that only a small fraction of the delivered cells are retained, especially in the long-term, with less than $1 \%$ of transplanted cells surviving in patient hearts. ${ }^{18}$ Because they are transplanted in an ischemic, hypoxic, and proapoptotic niche, most stem cells cannot survive after transplantation. Early apoptosis of the majority of stem cells after transplantation into the ischemic heart imposes restrictions on their repair functions. Thus, the challenge for cardiac cell therapy is to strengthen donor cells to allow survival and effective functioning in the harsh microenvironment of the infarcted heart. ${ }^{19}$

In response to these problems, we propose a regimen of combination drug therapy to enhance the therapeutic benefits of ASCs transplantation after AMI. As mentioned above, CsA is identified as an optimal drug due to its antiapoptotic properties, which are mediated through an inhibition of the mitochondrial apoptotic pathway. Recently, Dube et al ${ }^{20}$ reported the synthesis of a modified mitochondrial-targeted CsA which improved the cyclophilin-D-binding affinity and showed better cardioprotective effects than CsA. Importantly, our data were in agreement with those reported by Piot et al, ${ }^{5}$ who carried out a well-designed pilot study of CsA in patients with ST-segment elevation AMI, and demonstrated smaller infarct sizes after percutaneous coronary intervention, which was confirmed by DE-MRI. Mewton et $\mathrm{al}^{21}$ recently confirmed that CsA-induced reduction in infarct size was associated with a lower left ventricle dilatation at day 5 , which was maintained at 6 months.

However, serious side effects are associated with CsA clinical use, including renal toxicity, ${ }^{6}$ which dictates changes in therapeutic strategies, such as the development of alternative and less toxic CsA formulations with controlled drug release and targeting of CsA to the heart. Building on the successful targeting drug delivery system of cancer chemotherapy, the present work prepared and characterized CsA incorporated into CsA-NP. Such systems offer advantages, such as low toxicity, high drug payload, drug targeting, and controlled release.$^{8}$ Carriers such as NP may potentially modify the distribution of drugs in the body, and consequently, important adverse effects can be minimized or even abolished.

Our data were in line with those reported by Fujiwara et $\mathrm{al}_{, 2}{ }^{22}$ in which the appearance of beating colonies from human induced pluripotent stem cells was increased approximately 4.3 -fold by addition of CsA. These results provided a technological basis to obtain functional cardiomyocytes. The molecular mechanisms behind this potent CsA effect on the cardiac lineage remain unknown. Another calcineurin inhibitor, FK506, and a nuclear factor of activated T-cells inhibitor, 11R-VIVIT, have been examined but neither reproduced the effects of $\mathrm{CsA},{ }^{23}$ indicating that the CsA effect is mediated by a molecular mechanism other than immunosuppression.

In summary, we found that combination therapy with CsA-NP enhanced the viability of ASCs, improved LV global function recovery, and prevented LV volume expansion. These effects are possibly attributable to its antiapoptotic, 
prosurvival, proliferation-inducing, and neovascularizationpromoting effects. Taken together, these results ultimately could contribute to the development of an effective, simple, clinically applicable, and widely available protocol for myocardial regeneration. The beneficial effects of ASCs in combination with CsA-NP on LV function and remodeling documented in this experimental large animal model warrant further investigation to assess the therapeutic potential of this approach at the time of revascularization in patients with AMI.

\section{Acknowledgment}

This study was supported by the National High Technology Research and Development Program of China (2006AA02A105). The funders had no role in study design, data collection and analysis, decision to publish, or preparation of the manuscript. We also thank the anonymous reviewers for critical reading and discussions of the manuscript.

\section{Disclosure}

The authors report no conflicts of interest in this work. The data in this manuscript are original.

\section{References}

1. Shi CZ, Zhang XP, Lv ZW, et al. Adipose tissue-derived stem cells embedded with eNOS restore cardiac function in acute myocardial infarction model. Int J Cardiol. 2012;154(1):2-8.

2. De Ugarte DA, Morizono K, Elbarbary A, et al. Comparison of multilineage cells from human adipose tissue and bone marrow. Cells Tissues Organs. 2003;174(3):101-109.

3. Toma C, Pittenger MF, Cahill KS, Byrne BJ, Kessler PD. Human mesenchymal stem cells differentiate to a cardiomyocyte phenotype in the adult murine heart. Circulation. 2002;105(1):93-98.

4. Perrelli MG, Pagliaro P, Penna C. Ischemia/reperfusion injury and cardioprotective mechanisms: role of mitochondria and reactive oxygen species. World J Cardiol. 2011;3(6):186-200.

5. Piot C, Croisille P, Staat P, et al. Effect of cyclosporine on reperfusion injury in acute myocardial infarction. N Engl J Med. 2008;359(5): 473-481.

6. Tjia JF, Webber IR, Back DJ. Cyclosporin metabolism by the gastrointestinal mucosa. Br J Clin Pharmacol. 1991;31(3):344-346.

7. Zhang Y, Li X, Zhou Y, et al. Preparation and evaluation of poly(ethylene glycol)-poly(lactide) micelles as nanocarriers for oral delivery of cyclosporine a. Nanoscale Res Lett. 2010;5(6):917-925.
8. Singh S, Dobhal AK, Jain A, Pandit JK, Chakraborty S. Formulation and evaluation of solid lipid nanoparticles of a water soluble drug: Zidovudine. Chem Pharm Bull (Tokyo). 2010;58(5):650-655.

9. Yin QX, Pei ZY, Wang H, Zhao YS. [Pretreatment with cyclosporin A nanoparticles emulsion protects apoptosis of swine adipose tissuederived stem cells]. Zhonghua Xin Xue Guan Bing Za Zhi. 2013;41(6): 501-506. Chinese.

10. Monaco E, de Lima AS, Bionaz M, et al. Morphological and transcriptomic comparison of adipose and bone marrow derived porcine stem cells. J Tissue Eng Regen Med. 2009;2:20-33.

11. Lim SY, Hausenloy DJ, Arjun S, et al. Mitochondrial cyclophilin-D as a potential therapeutic target for post-myocardial infarction heart failure. J Cell Mol Med. 2011;15(11):2443-2451.

12. Malouitre S, Dube H, Selwood D, Crompton M. Mitochondrial targeting of cyclosporin A enables selective inhibition of cyclophilin-D and enhanced cytoprotection after glucose and oxygen deprivation. Biochem $J$. 2010;425(1):137-148.

13. Onishi A, Miyamae M, Kaneda K, Kotani J, Figueredo VM. Direct evidence for inhibition of mitochondrial permeability transition pore opening by sevoflurane preconditioning in cardiomyocytes: comparison with cyclosporine A. Eur J Pharmacol. 2012;675(1-3):40-46.

14. Jameel MN, Zhang J. Stem cell therapy for ischemic heart disease. Antioxid Redox Signal. 2010;13(12):1879-1897.

15. Monaco E, Bionaz M, Rodriguez-Zas S, Hurley WL, Wheeler MB. Transcriptomics comparison between porcine adipose and bone marrow mesenchymal stem cells during in vitro osteogenic and adipogenic differentiation. PLoS One. 2012;7(3):e32481.

16. Madonna R, De Caterina R. Adipose tissue: a new source for cardiovascular repair. J Cardiovasc Med (Hagerstown). 2010;11(2): $71-80$.

17. Duckers MD. Treating heart attack with fat-derived stem cells may be safe in humans be safe in humans. In: American Heart Association Meeting Report; November 16, 2010; Chicago, IL. Abstract 12225.

18. Schoenhard JA, Hatzopoulos AK. Stem cell therapy: pieces of the puzzle. J Cardiovasc Transl Res. 2010;3(1):49-60.

19. Moreira Rde C, Haddad AF, Silva SA, et al. Intracoronary stem-cell injection after myocardial infarction: microcirculation sub-study. Arq Bras Cardiol. 2011;97(5):420-426.

20. Dube H, Selwood D, Malouitre S, Capano M, Simone MI, Crompton M. A mitochondrial-targeted cyclosporin A with high binding affinity for cyclophilin D yields improved cytoprotection of cardiomyocytes. Biochem J. 2012;441(3):901-907.

21. Mewton N, Croisille P, Gahide G, et al. Effect of cyclosporine on left ventricular remodeling after reperfused myocardial infarction. $J \mathrm{Am}$ Coll Cardiol. 2010;55(12):1200-1205.

22. Fujiwara M, Yan P, Otsuji TG, et al. Induction and enhancement of cardiac cell differentiation from mouse and human induced pluripotent stem cells with cyclosporin-A. PLoS One. 2011;6(2):e16734.

23. Yan P, Nagasawa A, Uosaki H, et al. Cyclosporin-A potently induces highly cardiogenic progenitors from embryonic stem cells. Biochem Biophys Res Commun. 2009;379(1):115-120.
International Journal of Nanomedicine

\section{Publish your work in this journal}

The International Journal of Nanomedicine is an international, peerreviewed journal focusing on the application of nanotechnology in diagnostics, therapeutics, and drug delivery systems throughout the biomedical field. This journal is indexed on PubMed Central, MedLine, CAS, SciSearch $\AA$, Current Contents ${ }^{\circledR} /$ Clinical Medicine,

\section{Dovepress}

Journal Citation Reports/Science Edition, EMBase, Scopus and the Elsevier Bibliographic databases. The manuscript management system is completely online and includes a very quick and fair peer-review system, which is all easy to use. Visit http://www.dovepress.com/ testimonials.php to read real quotes from published authors. 